Cronfa - Swansea University Open Access Repository

This is an author produced version of a paper published in :

Physiological Measurement

Cronfa URL for this paper:

http://cronfa.swan.ac.uk/Record/cronfa21301

\title{
Paper:
}

Lewis, M. (2015). Changes in Heart Rate Variability and QT Variability during the First Trimester of Pregnancy. Physiological Measurement

This article is brought to you by Swansea University. Any person downloading material is agreeing to abide by the terms of the repository licence. Authors are personally responsible for adhering to publisher restrictions or conditions. When uploading content they are required to comply with their publisher agreement and the SHERPA RoMEO database to judge whether or not it is copyright safe to add this version of the paper to this repository. http://www.swansea.ac.uk/iss/researchsupport/cronfa-support/ 


\section{Changes in Heart Rate Variability and QT Variability during the First Trimester of Pregnancy}

RE Carpenter ${ }^{1}$, LA D'Silva ${ }^{1}$, SJ Emery ${ }^{2}$, O Uzun $^{3}$, D Rassi ${ }^{4}$, MJ Lewis ${ }^{1 *}$

${ }^{1}$ College of Engineering, Swansea University, Swansea, UK

${ }^{2}$ Department of Gynaecology, Singleton Hospital, Swansea, UK

${ }^{3}$ Department of Paediatric Cardiology, University Hospital of Wales, Cardiff, UK

${ }^{4}$ College of Health and Human Sciences, Swansea University, Swansea, UK

${ }^{*}$ Corresponding author address:

College of Engineering

Swansea University

Talbot Building

Singleton Park

Swansea

SA2 8PP

Tel. +44 1792513043

Email. m.j.lewis@swansea.ac.uk

Keywords: Heart rate variability, QT variability, Pregnancy, Arrhythmia, Autonomic nervous system

Short Title: Early Cardiovascular Changes during Pregnancy 


\section{Abstract}

The risk of new-onset arrhythmia during pregnancy is high, presumably relating to changes in both haemodynamic and cardiac autonomic function. The ability to non-invasively assess an individual's risk of developing arrhythmia during pregnancy would therefore be clinically significant. We aimed to quantify electrocardiographic temporal characteristics during the first trimester of pregnancy and to compare these with non-pregnant controls.

Ninety-nine pregnant women and sixty-three non-pregnant women underwent non-invasive cardiovascular and haemodynamic assessment during a protocol consisting of various physiological states (postural manoeurvres, light exercise and metronomic breathing). Variables measured included stroke volume, cardiac output, heart rate, heart rate variability, QT and QT variability and QTVI (a measure of the variability of QT relative to that of RR).

Heart rate $(p<0.0005, p<0.0005, p<0.0005)$ and cardiac output $(p=0.043, p<0.0005$, $p<0.0005$ ) were greater in pregnant women in all physiological states (respectively for the supine position, light exercise and metronomic breathing state), whilst stroke volume was lower in pregnancy only during the supine position $(p<0.0005)$. QTe ( $Q$ wave onset to $T$ wave end) and QTa (T wave apex) were significantly shortened $(p<0.05)$ and QTeVI and QTaVI were increased in pregnancy in all physiological states $(p<0.0005)$. QT variability $(p<0.002)$ was greater in pregnant women during the supine position, whilst heart rate variability was reduced in pregnancy in all states $(p<0.0005)$.

Early pregnancy is associated with substantial changes in heart rate variability, reflecting a reduction in parasympathetic tone and an increase in sympathetic activity. QTVI shifted to a less favourable value, reflecting a greater than normal amount of QT variability. QTVI appears to be a useful method for quantifying changes in QT variability relative to RR (or heart rate) variability, being sensitive not only to physiological state but also to gestational age. We support the use of non-invasive markers of cardiac electrical variability to evaluate the risk of arrhythmic events in pregnancy, and we recommend the use of multiple physiological states during the assessment protocol. 


\section{Introduction}

Women tend to have higher intrinsic heart rates (Jose \& Collison, 1970) and more prolonged QT intervals (Molnar et al., 1996) compared with men. Some cardiac arrhythmias are also more prevalent in women, such as the increased incidence of supraventricular tachycardias (SVT) (Porter et al., 2004). The risk of new onset SVT is further elevated during normal pregnancy (Kron \& Conti, 2007) with 24 per 100,000 pregnancies experiencing SVT (Li et al., 2008), as is the incidence of symptomatic ectopic activity, with up to $50 \%$ of pregnant women experiencing non-sustained ventricular arrhythmias at some point during their pregnancy (Kron \& Conti, 2007). The incidence of pregnancy-induced arrhythmia appears to be uniform throughout pregnancy with perhaps an increased frequency during late pregnancy (Shotan et al., 1997; Nakagawa et al., 2004), although information on this is limited. This elevated incidence is presumably related to changes in both cardiac autonomic function (for supraventricular events) and in ion channel conduction (for ventricular events) within the myocardium. Pregnancy is also associated with substantial haemodynamic changes, which can cause myocardial stretching and may thus promote arrhythmogenic events (Kanoupakis \& Vardas, 2005; Adamson \& Nelson-Piercy, 2007). The ability to noninvasively assess (and act upon) an individual's risk of developing arrhythmia during pregnancy would therefore be clinically significant.

Changes in autonomic activity during pregnancy are poorly documented (Kuo et al., 2000). The general consensus is that heart rate variability (HRV, a surrogate marker of cardiac autonomic control that describes beat-to-beat variation in cardiac (RR) interval) decreases with advancing gestation (Speranza et al., 1998; Kuo et al., 2000; Chamchad et al., 2007). However, these findings are mainly based on measurements taken during late pregnancy and then compared with non-pregnant controls. The extent of changes in cardiac autonomic function during earlier stages of pregnancy is not known, but marked increases in sympathetic activity have been observed in other body systems within the first few weeks of conception (Jarvis et al., 2012). Clearly a comprehensive description of normal cardiac autonomic changes from the earliest stages of pregnancy would be necessary in the context of developing a biomarker of antenatal arrhythmogenic risk (Nakagawa et al., 2004). 
The QT interval is a measure of ventricular depolarisation and repolarisation within the cardiac cycle, which is strongly influenced by heart rate as well other chronotropic factors. The QT interval has not been extensively studied during pregnancy. QT interval shortens during the second-half of pregnancy and remains reduced for up to three days post-partum, reflecting its heart rate-dependence (Baumert et al., 2010). The QT variability index (QTVI; Berger et al., (1997)) extends the concept of HRV to a comparison of the relative magnitudes of temporal variability within the electrocardiographic RR and QT intervals. QTVI is a good indicator of elevated risk for ventricular arrhythmic events - for example, QTVI is associated with an independent risk for ventricular tachycardia or ventricular fibrillation events (Haigney et al., 2004). QTVI has not been assessed during pregnancy, although Baumert et al. (2010) observed that QT variability is higher by late pregnancy (post 28 weeks gestation) in comparison with non-pregnant women.

The aim of this study was to quantify changes in RR and QT variabilities (including QTVI) during relatively early stages of pregnancy (the end of the first trimester) in the context of assessing their use as possible biomarkers for antenatal maternal arrhythmia risk.

\section{Method}

\subsection{Participants}

2.1.1 Non-pregnant: Eligible participants were women aged between 18 and 40 years, were not pregnant at the time of measurement, and had no history of cardiovascular or chronic respiratory disease, sleep apnoea or central or peripheral nervous system disorders. Written informed consent was taken at the initial meeting and participants were provided with a participant information sheet. Ethical approval was obtained from the College of Engineering's Applied Sport and Technology Exercise and Medicine (A-STEM) Ethics Committee and all procedures were conducted in accordance with the Declaration of Helsinki.

2.1.2 Pregnant: Eligible participants were apparently healthy pregnant women aged 18 years or over, with no existing complications of pregnancy at their 12-week dating scan. Participants were recruited via a number of methods (via direct contact at the antenatal 
clinic, strategically placed posters, advertisements in local newspapers and via email). Exclusion criteria were: a history of cardiovascular or chronic respiratory problems, sleep apnoea, or a central/peripheral nervous system disorder. Recruited participants were provided with details about the study, including practical requirements and potential risks, and were given one week to consider whether they wished to take part. Individuals who wanted to participate gave their written consent. Participants were informed that they were free to leave the study at any time and this would not affect their standard antenatal care. Ethical approval was obtained from the local (South West Wales) Research Ethics Committee and all procedures were conducted in accordance with the Declaration of Helsinki.

\subsection{Physiological measurements}

HRV can be quantified reproducibly during rest and exercise (McNarry \& Lewis, 2012) and our previous observations indicate that this is also generally true for beat-to-beat haemodynamic variables (D'Silva et al., 2014). Consequently single physiological assessments were considered appropriate at each defined stage of the assessment schedule. Physiological monitoring was carried out on four occasions for pregnant participants: at 12-16, $24-26$ and 34-36 weeks gestational age, corresponding to the end of the three trimesters (T1, T2, T3) and also at 12-weeks post-partum (PP). Non-pregnant participants underwent physiological assessment on one occasion only. All participants were asked to perform a series of postural manoeuvres and various interventions designed to provoke changes in the cardiovascular and autonomic nervous systems. Participants were asked to refrain from drinking tea, coffee, alcohol or a heavy meal within 2 hours prior to assessment and to not exercise within 24 hours prior to assessment.

\subsubsection{Experimental protocol: Participants were first asked to lie in a $45^{\circ}$ reclined-supine} position for six minutes, after which they were asked to stand for the same duration. Participants then performed a light stepping exercise for six minutes, using the Nintendo Wii games console and 'balance board' platform (to provide a visual stimulus for exercise). This was followed by a six minute seated recovery period. Participants then undertook a three minute cognitive test in the seated position (to provoke a sympathetic autonomic response), during which they were asked to repeatedly subtract the number seventeen from a four 
digit number. The arithmetic test was carried out in the participant's head (they did not need to give a verbal answer). Participants then breathed in time to a metronome for three minutes at a rate of 20 breaths per minute (designed to initiate a parasympathetic response) and then to return to their normal (spontaneous) breathing pattern for three minutes. The total duration of the measurement protocol was thirty-three minutes.

\subsubsection{Physiological variables quantified: Participants underwent continuous Holter ECG} monitoring (Pathfinder/Lifecard Digital system; Spacelabs Medical Ltd., UK), providing ECG data with a $1024 \mathrm{~Hz}$ sampling frequency. The ECG recordings were assessed for quality by human observation using the Pathfinder system, primarily to verify the absence of excessive noise or artefact. Beat-to-beat cardiac interval (RR) was measured automatically by the Pathfinder system (using a proprietary algorithm) and visually assessed to identify and delete any obvious artefacts (which occurred infrequently, with less than $0.1 \%$ of beats edited in this way). Heart rate variability (HRV) was quantified in terms of RMSSD (square root of the mean squared differences in successive RR intervals), SDRR (standard deviation of the RR intervals), LF (low frequency power), HF (high frequency power), VLF (very low frequency power), LFn (normalised low-frequency component), HFn (normalised highfrequency component) and Total Power.

QTe ( $Q$ wave onset to $T$ wave end), QTa ( $Q$ wave onset to T wave apex) and RR intervals were measured for each sinus beat and exported for further analysis using the Reynolds Research Tools software (Del Mar Reynolds Medical Ltd., UK). QTeVI and QTaVI were calculated according to the equation described by Berger et al (1997):

$$
\mathrm{QTVI}=\log _{10}\left[\left(\mathrm{QTV} / \mathrm{QTm}{ }^{2}\right) /\left(\mathrm{RRv} / \mathrm{RRm}^{2}\right)\right]
$$

where QTv and RRv are the variabilities of QT and RR, respectively, and QTm and RRm are the mean values of these parameters for each defined period.

RMSSDQT (square root of the mean squared differences in successive QT intervals) and SDQT (standard deviation of the QT intervals) were also calculated. The Task Force Haemodynamic monitor (CNSystems Medizintechnik GMBH, Austria) recorded beat-to-beat cardiovascular performance via impedance cardiography (transthoracic bioelectrical impedance analysis) and beat-to-beat systolic and diastolic blood pressures (SBP, DBP) via 
vascular unloading photoplethysmography. The following variables were also determined; heart rate (HR), stroke volume (SV), cardiac output (CO), total peripheral resistance (TPR), compliance, stiffness, left ventricular ejection time (LVET) \& end diastolic index (EDI).

\subsection{Statistical Analysis}

Normality of the data was assessed using the Kolmogorov-Smirnov test. Independent samples t-tests were used to assess between group differences at each stage of the protocol. Data are presented for the non-pregnant participants (NP) and for the first antenatal measurement (T1). Results for the gestational changes will be presented elsewhere. Statistical significance was accepted as $p<0.05$. All data are presented as Mean \pm SEM (standard error of the mean).

\section{Results}

\subsection{Participant characteristics}

Ninety-nine pregnant women completed the first antenatal measurement (T1) at a mean gestational age of $15.1 \pm 1.7$ weeks and sixty-three non-pregnant participants were assessed. Participant characteristics are displayed in Table 1. 49.5\% of pregnant women were nulliparous and $50.5 \%$ primiparous or multiparous.

Table 1. Participant characteristics (mean \pm SD)

\begin{tabular}{ccc}
\hline & $\begin{array}{c}\text { Non-pregnant } \\
(\mathrm{n}=63)\end{array}$ & $\begin{array}{c}\text { T1 } \\
(\mathrm{n}=99)\end{array}$ \\
\hline Age (years) & $23.5 \pm 5.6$ & $29.1 \pm 4.9$ \\
Height $(\mathrm{cm})$ & $167.1 \pm 6.7$ & $164.0 \pm 5.3$ \\
Weight $(\mathrm{kg})$ & $63.9 \pm 9.8$ & $68.8 \pm 14.1$ \\
BMI $\left(\mathrm{kg} \cdot \mathrm{m}^{-2}\right)$ & $22.9 \pm 2.9$ & $25.6 \pm 5.2$ \\
\hline
\end{tabular}

\subsection{Haemodynamics}

Figure 1 compares the HR, SV, CO and SBP for pregnant and non-pregnant groups during the supine, exercise and metronomic breathing states. In summary: (1) HR ( $p<0.0005, p<0.0005$, $p<0.0005$ respectively for physiological state $)$ and $C O(p=0.043, p<0.0005, p<0.0005)$ were 
significantly greater in pregnant women than in non-pregnant controls in all three states; (2) SV was lower during pregnancy but only in the supine position $(p<0.0005)$; (3) TPR was lower during pregnancy in all states $(p=0.004, p<0.0005, p<0.0005) ;(4)$ Blood pressure (MBP, SBP, DBP) $(p=0.010, p=0.008, p=0.011)$ and EDI $(p<0.001)$ were significantly reduced in pregnancy only during metronomic breathing; $(5)$ Compliance $(p=0.001), E D I(p<0.0005)$ and LVET $(p<0.0005)$ were all reduced in pregnancy but only in the supine position (Table 2 ).

a.)

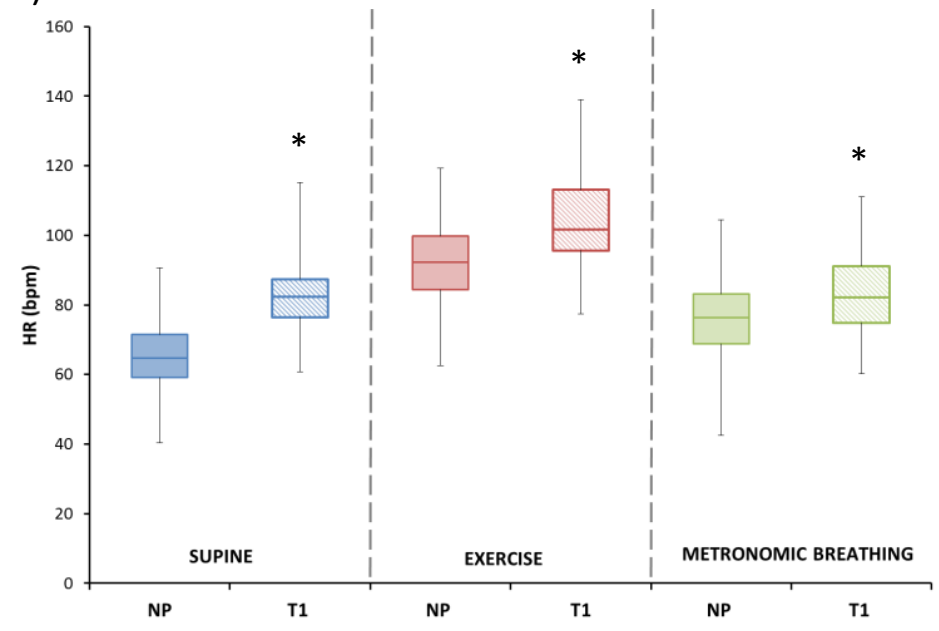

c.)

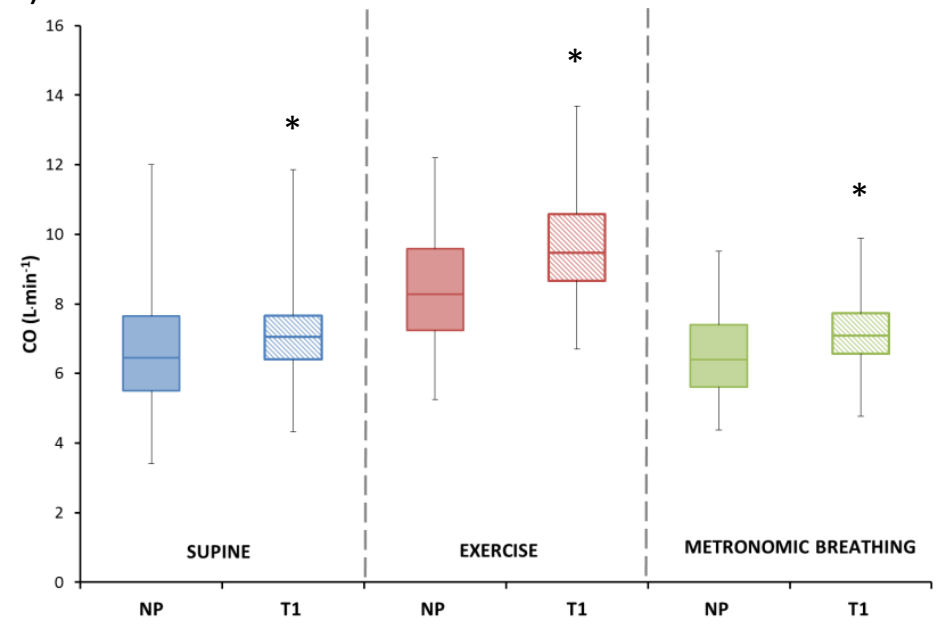

b.)

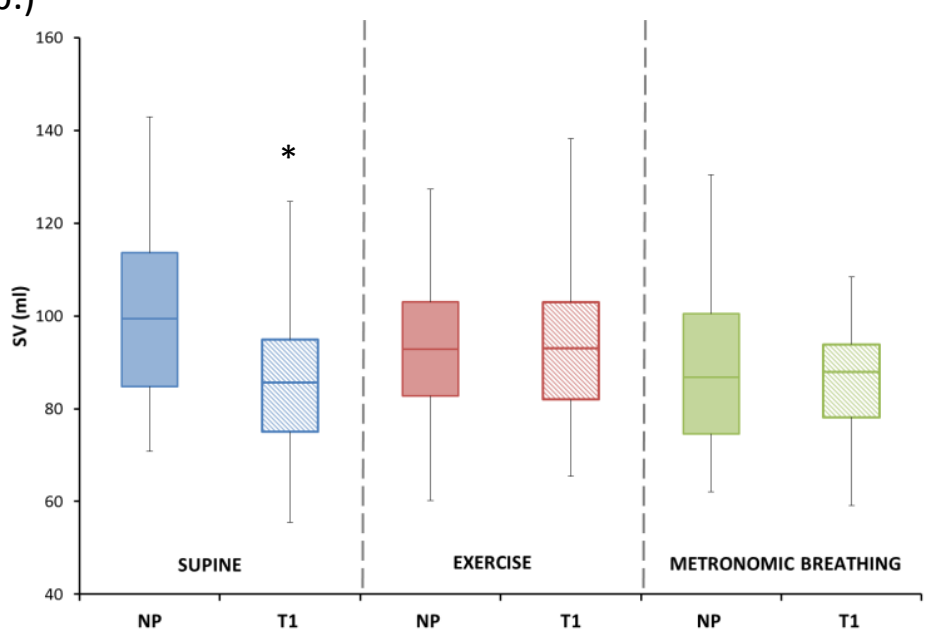

d.)

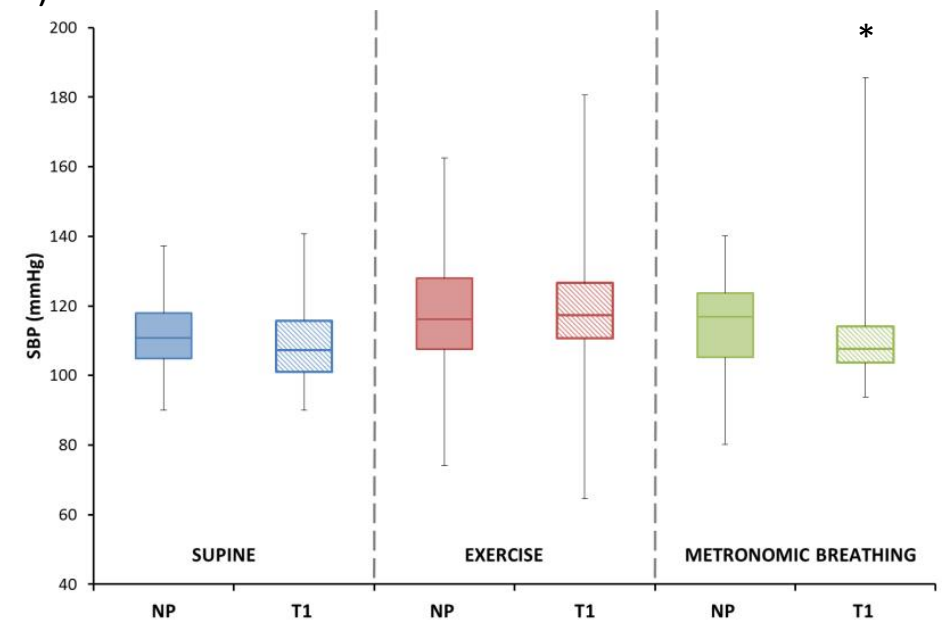

${ }^{*}$ Statistically different from non-pregnant values.

Figure 1. Haemodynamic variables during the supine, exercise and metronomic breathing states for pregnant and non-pregnant groups. Box plots display the median and upper/lower quartiles of the data, whilst the whiskers show the maximum and minimum values. Solid boxes represent non-pregnant participants and shaded bars represent pregnant participants. 


\subsection{QT Variability}

Figure 2 compares QTe, RMSSDQTe, SDQTe and QTeVI for pregnant and non-pregnant groups during the supine, exercise and metronomic breathing states. All QTa variables can be found in Table 3. In summary: (1) QTe and QTa were significantly shortened in pregnant women than in non-pregnant controls in the supine position $(p<0.0005, p<0.0005)$, during exercise $(p=0.013, p=0.012)$ and during metronomic breathing $(p=0.001, p=0.001) ;(2)$ QTeVI and QTaVI were significantly increased (became less negative) with pregnancy in all physiological states ( $p<0.0005$ ); (3) RMSSDQTe and SDQTe were significantly greater in pregnant women than in non-pregnant controls but only in the supine position $(p<0.0005$, $p=0.002)$; (4) RMSSDQTa was greater in the supine posture $(p=0.009)$ and reduced during exercise $(p<0.0005)$ (Table 3$)$.

\subsection{Heart Rate Variability}

Figure 3 compares the RMSSD, SDRR, HFn, LFn and Total Power for pregnant and nonpregnant groups during the supine, exercise and metronomic breathing states. In summary: (1) RR ( $p<0.0005)$, RMSSD ( $<<0.0005)$, SDRR $(p<0.0005)$, Total Power $(p<0.0005, p=0.014$, $p<0.0005), H F(p<0.0005, p=0.002, p<0.0005)$, and LF ( $p<0.0005)$ (Table 4) were lower in pregnant women than in non-pregnant controls in all three states; (2) LFn was higher during pregnancy but only in the supine position ( $p<0.0005$ ); (3) HFn and VLF were lower during pregnancy in the supine position ( $p<0.0005, p<0.0005$ respectively) and during metronomic breathing ( $p=0.015, p<0.0005$ respectively) (Table 4). 
a.)

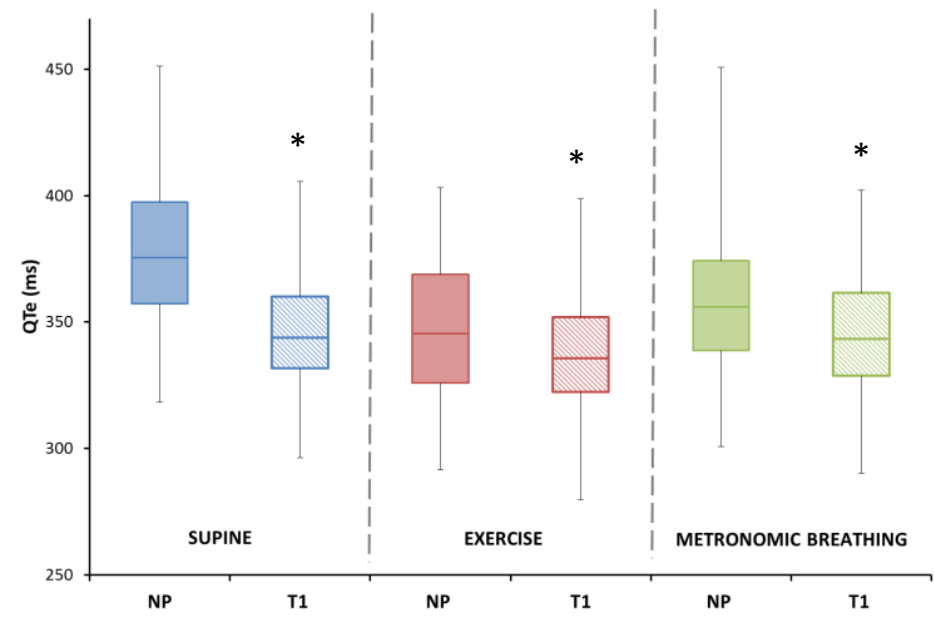

c.)

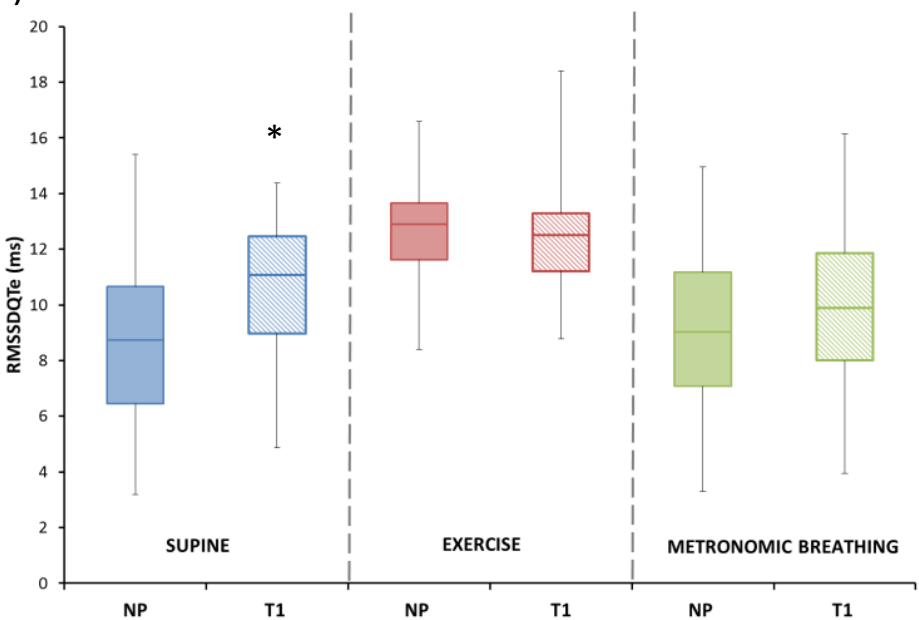

b.)

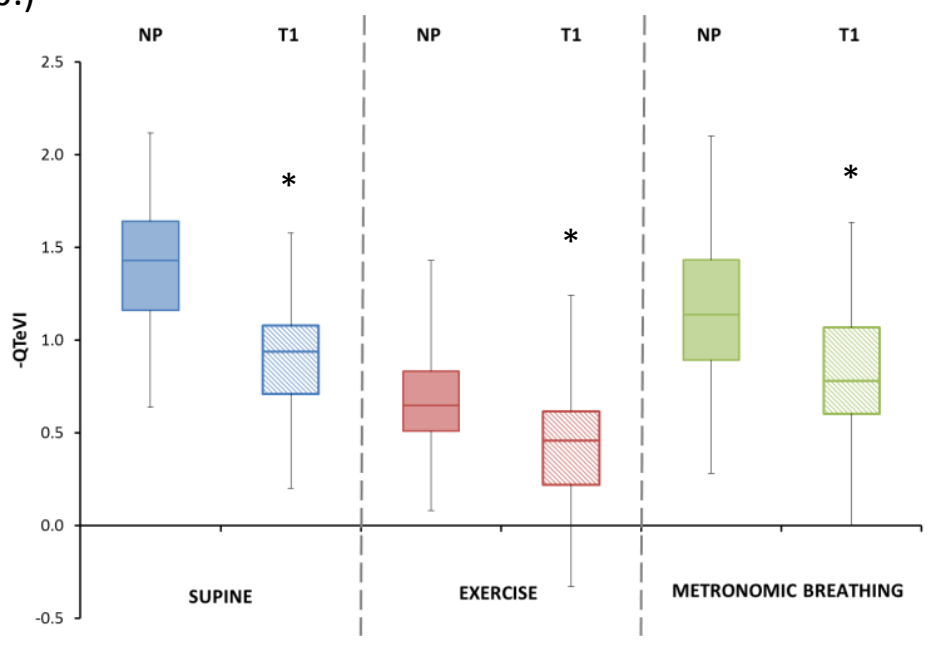

d.)

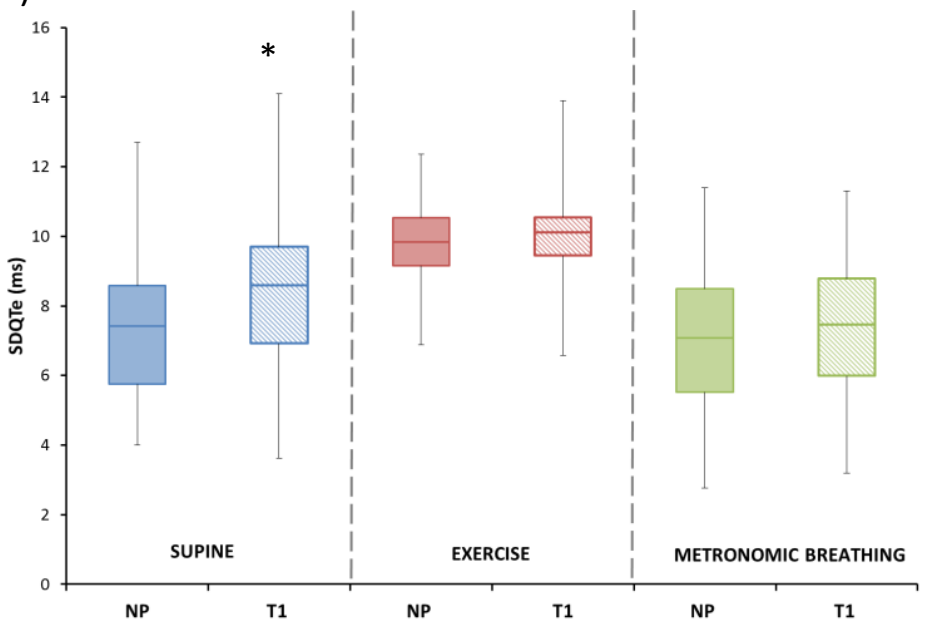

${ }^{*}$ Statistically different from non-pregnant values.

Figure 2. QT variables during supine, exercise and metronomic breathing states for pregnant and non-pregnant groups. Box plots display the median and upper/lower quartiles of the data, whilst the whiskers show the maximum and minimum values. Solid boxes represent non-pregnant participants and shaded bars represent pregnant participants. 
a.)

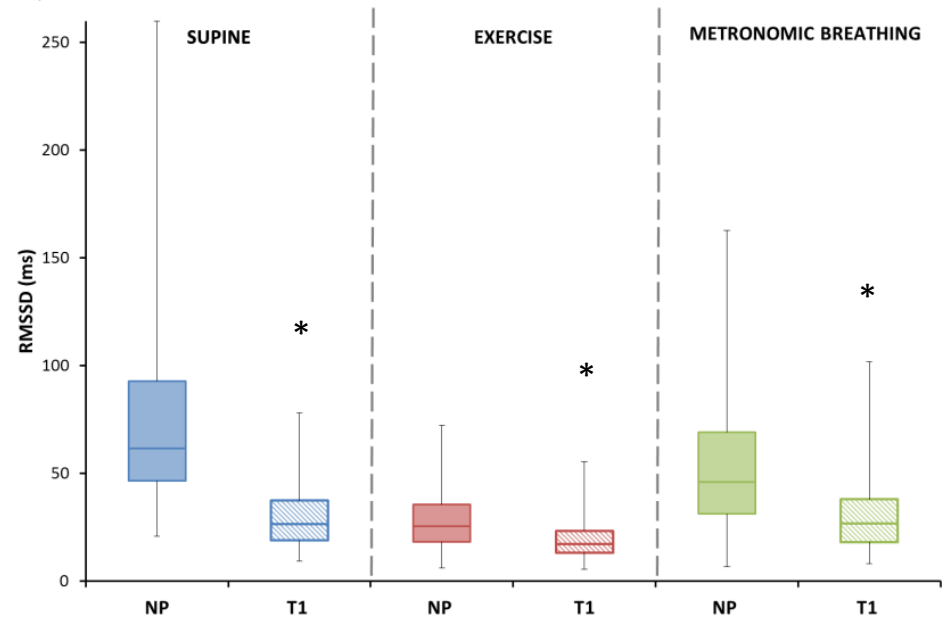

c.)

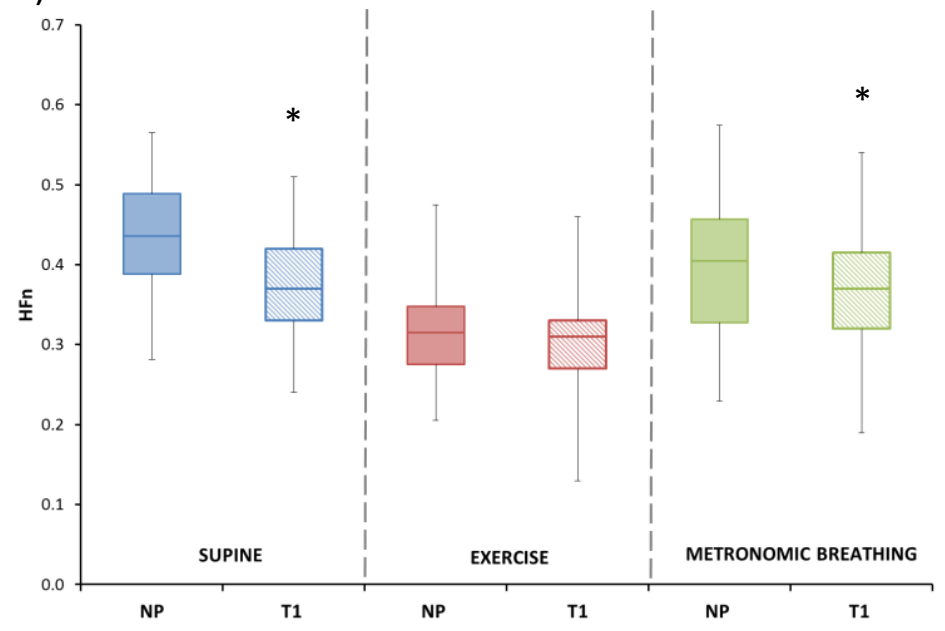

e.)

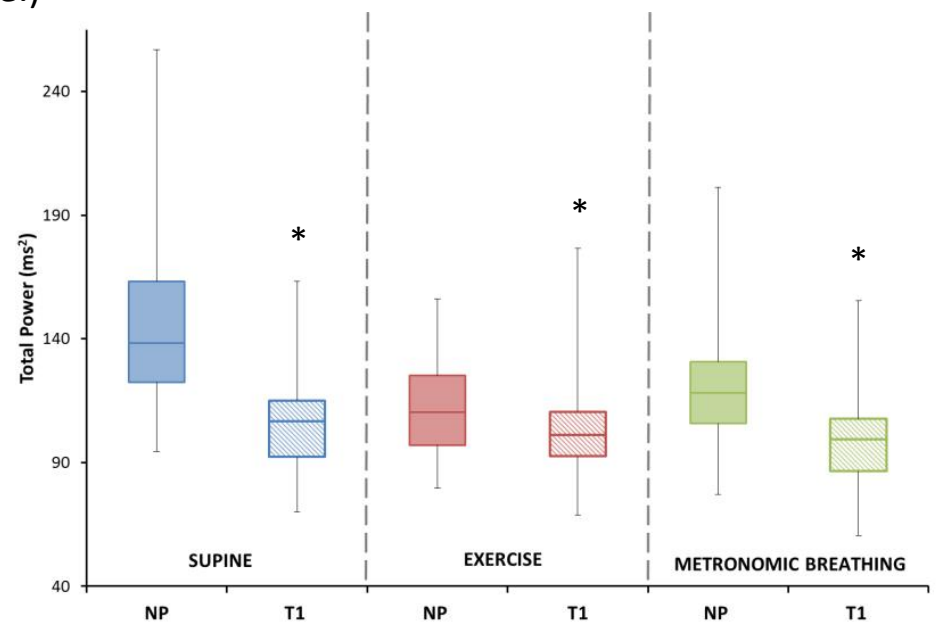

b.)

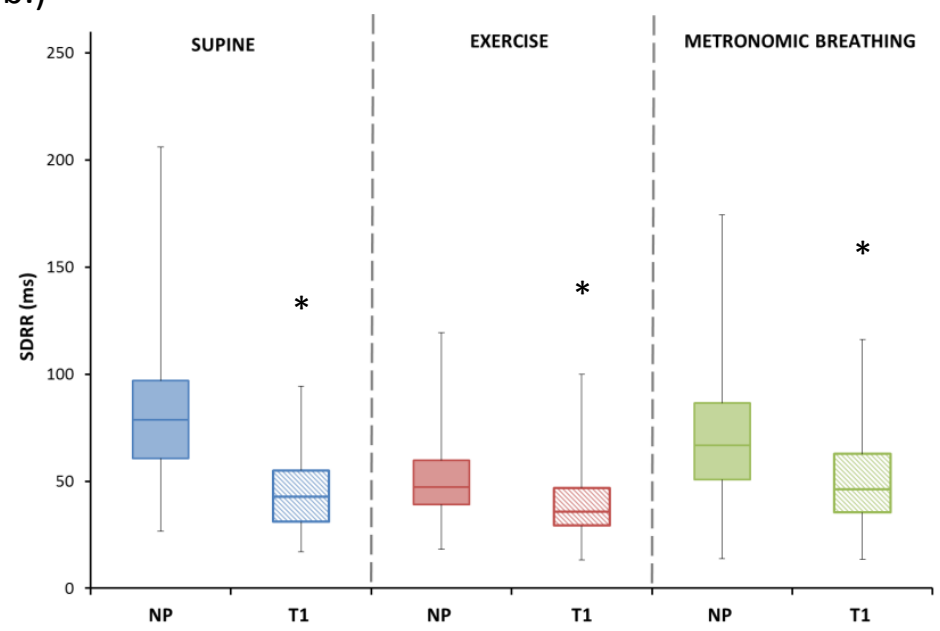

d.)

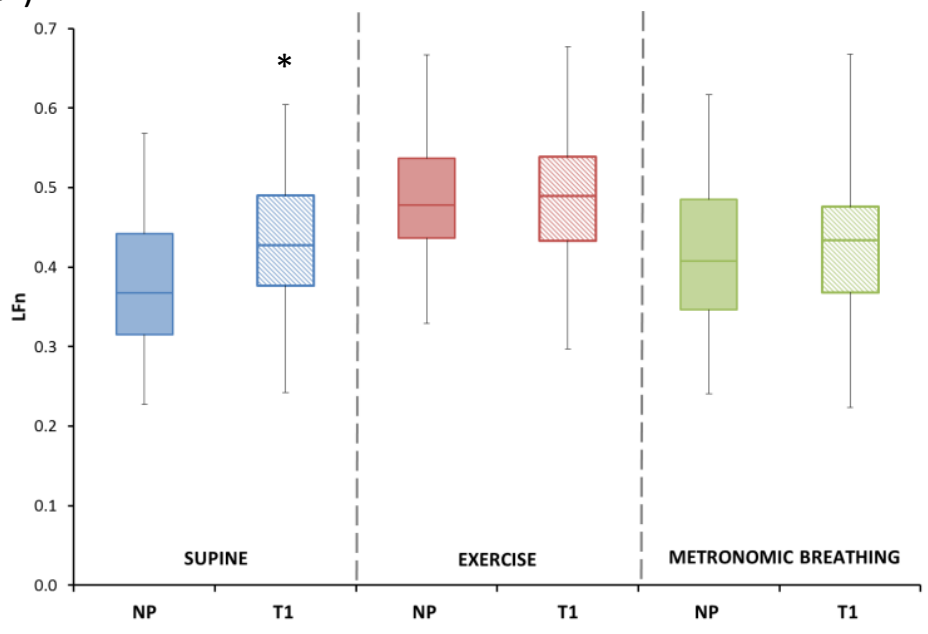

*Statistically different from non-pregnant values.

Figure 3. Heart Rate Variability variables during the supine, exercise and metronomic breathing states for pregnant and non-pregnant groups. Box plots display the median and upper/lower quartiles of the data, whilst the whiskers show the maximum and minimum values. Solid boxes represent non-pregnant participants and shaded bars represent pregnant participants. 
Table 2. Haemodynamic variables during the supine, exercise and metronomic breathing states for pregnant and non-pregnant groups (mean \pm SEM).

\begin{tabular}{|c|c|c|c|c|c|c|c|c|c|}
\hline & \multicolumn{3}{|c|}{ Supine } & \multicolumn{3}{|c|}{ Exercise } & \multicolumn{3}{|c|}{ Metronomic Breathing } \\
\hline & NP & $\mathrm{T} 1$ & $\mathrm{p}$ & NP & $\mathrm{T} 1$ & $\mathrm{p}$ & NP & $\mathrm{T} 1$ & $\mathrm{p}$ \\
\hline HR (bpm) & $65.5 \pm 1.3$ & $83.0 \pm 1.0$ & $*$ & $91.6 \pm 1.4$ & $103.8 \pm 1.3$ & $*$ & $75.1 \pm 1.5$ & $84.1 \pm 1.1$ & $*$ \\
\hline $\mathrm{SV}(\mathrm{ml})$ & $101.4 \pm 2.4$ & $86.2 \pm 1.5$ & $*$ & $93.4 \pm 2.0$ & $93.2 \pm 1.3$ & 0.924 & $88.7 \pm 2.0$ & $85.9 \pm 1.1$ & 0.228 \\
\hline $\mathrm{CO}\left(\mathrm{L} \cdot \mathrm{min}^{-1}\right)$ & $6.6 \pm 0.2$ & $7.1 \pm 0.1$ & 0.043 & $8.5 \pm 0.2$ & $9.6 \pm 0.1$ & $*$ & $6.6 \pm 0.1$ & $7.2 \pm 0.1$ & $*$ \\
\hline $\begin{array}{l}\text { TPR } \\
\left(\text { dyn.sec.cm }{ }^{-5}\right)\end{array}$ & $1025 \pm 32$ & $915 \pm 18$ & 0.004 & $839 \pm 21$ & $721 \pm 13$ & $*$ & $1047 \pm 23$ & $906 \pm 15$ & $*$ \\
\hline $\begin{array}{l}\text { Compliance } \\
\left(\mathrm{ml} \cdot \mathrm{mmHg}^{-1}\right)\end{array}$ & $2.6 \pm 0.1$ & $2.3 \pm 0.05$ & 0.001 & $2.2 \pm 0.1$ & $2.0 \pm 0.05$ & 0.107 & $2.1 \pm 0.1$ & $2.2 \pm 0.04$ & 0.608 \\
\hline $\begin{array}{l}\text { Stiffness } \\
\left(\mathrm{mmHg} \cdot \mathrm{ml}^{-1}\right)\end{array}$ & $0.4 \pm 0.01$ & $0.5 \pm 0.01$ & 0.001 & $0.5 \pm 0.02$ & $0.5 \pm 0.01$ & 0.105 & $0.5 \pm 0.01$ & $0.5 \pm 0.01$ & 0.608 \\
\hline $\mathrm{SBP}(\mathrm{mmHg})$ & $111.5 \pm 1.3$ & $108.5 \pm 1.0$ & 0.065 & $117.5 \pm 2.2$ & $118.9 \pm 1.6$ & 0.524 & $115.2 \pm 1.4$ & $110.4 \pm 1.2$ & 0.008 \\
\hline $\mathrm{DBP}(\mathrm{mmHg})$ & $71.0 \pm 1.0$ & $69.8 \pm 0.8$ & 0.345 & $71.7 \pm 1.4$ & $70.4 \pm 1.1$ & 0.454 & $72.8 \pm 1.0$ & $69.6 \pm 0.9$ & 0.011 \\
\hline $\mathrm{MBP}(\mathrm{mmHg})$ & $83.0 \pm 1.1$ & $81.5 \pm 0.9$ & 0.299 & $87.1 \pm 1.6$ & $86.6 \pm 1.2$ & 0.807 & $86.2 \pm 1.1$ & $82.5 \pm 1.0$ & 0.010 \\
\hline LVET (ms) & $319.4 \pm 2.1$ & $290.2 \pm 1.8$ & $*$ & $271.0 \pm 2.0$ & $255.3 \pm 1.6$ & $*$ & $287.6 \pm 2.9$ & $282.9 \pm 2.0$ & 0.169 \\
\hline $\mathrm{EDI}\left(\mathrm{ml} \cdot \mathrm{m}^{-2}\right)$ & $93.9 \pm 1.9$ & $79.8 \pm 1.3$ & $*$ & $92.9 \pm 1.4$ & $91.2 \pm 1.1$ & 0.350 & $88.5 \pm 1.5$ & $82.3 \pm 1.1$ & 0.001 \\
\hline
\end{tabular}

$* \mathrm{p}<5 \times 10^{-4}$

Table 3. QT variables during the supine, exercise and metronomic breathing states pregnant and non-pregnant groups (mean \pm SEM).

\begin{tabular}{l|ccc|cccccc}
\hline & \multicolumn{3}{|c}{ Supine } & \multicolumn{3}{c|}{ Exercise } & \multicolumn{3}{c}{ Metronomic Breathing } \\
& NP & T1 & $\mathrm{p}$ & $\mathrm{NP}$ & $\mathrm{T} 1$ & $\mathrm{p}$ & $\mathrm{NP}$ & $\mathrm{T} 1$ & $\mathrm{p}$ \\
\hline QTe (ms) & $376.4 \pm 3.8$ & $346.3 \pm 2.3$ & $*$ & $347.2 \pm 3.3$ & $337.3 \pm 2.2$ & 0.013 & $358.9 \pm 3.9$ & $344.4 \pm 2.3$ & 0.001 \\
QTeVI & $-1.4 \pm 0.04$ & $-0.9 \pm 0.03$ & $*$ & $-0.7 \pm 0.04$ & $-0.4 \pm 0.03$ & $*$ & $-1.1 \pm 0.05$ & $-0.8 \pm 0.04$ & $*$ \\
RMSSDQTe (ms) & $8.9 \pm 0.4$ & $10.6 \pm 0.2$ & $*$ & $12.6 \pm 0.2$ & $12.3 \pm 0.2$ & 0.322 & $9.2 \pm 0.4$ & $9.9 \pm 0.3$ & 0.120 \\
SDQTe (ms) & $7.4 \pm 0.3$ & $8.4 \pm 0.2$ & 0.002 & $9.8 \pm 0.2$ & $10.1 \pm 0.1$ & 0.053 & $7.2 \pm 0.3$ & $7.5 \pm 0.2$ & 0.361 \\
QTa (ms) & $312.4 \pm 3.5$ & $284.5 \pm 2.1$ & $*$ & $286.7 \pm 2.7$ & $278.3 \pm 1.9$ & 0.012 & $295.8 \pm 3.5$ & $282.7 \pm 2.1$ & 0.001 \\
QTaVI & $-1.3 \pm 0.04$ & $-0.9 \pm 0.03$ & $*$ & $-0.7 \pm 0.04$ & $-0.4 \pm 0.03$ & $*$ & $-1.0 \pm 0.04$ & $-0.7 \pm 0.03$ & $*$ \\
RMSSDQTa (ms) & $8.6 \pm 0.2$ & $9.3 \pm 0.2$ & 0.009 & $10.6 \pm 0.2$ & $9.8 \pm 0.1$ & $*$ & $9.5 \pm 0.2$ & $9.3 \pm 0.2$ & 0.475 \\
SDQTa (ms) & $6.8 \pm 0.2$ & $7.2 \pm 0.2$ & 0.065 & $8.3 \pm 0.1$ & $8.4 \pm 0.1$ & 0.481 & $7.1 \pm 0.2$ & $7.0 \pm 0.2$ & 0.564 \\
\hline
\end{tabular}

$* p<5 \times 10^{-4}$ 
Table 4. Heart rate variability indices during the supine, exercise and metronomic breathing states pregnant and non-pregnant groups (mean $\pm \mathrm{SEM}$ ).

\begin{tabular}{|c|c|c|c|c|c|c|c|c|c|}
\hline & \multicolumn{3}{|c|}{ Supine } & \multicolumn{3}{|c|}{ Exercise } & \multicolumn{3}{|c|}{ Metronomic Breathing } \\
\hline & NP & $\mathrm{T} 1$ & $\mathrm{p}$ & NP & $\mathrm{T} 1$ & $\mathrm{p}$ & NP & $\mathrm{T} 1$ & $\mathrm{p}$ \\
\hline RMSSD (ms) & $77.0 \pm 6.3$ & $29.9 \pm 1.5$ & $*$ & $28.3 \pm 1.6$ & $19.3 \pm 0.9$ & $*$ & $53.4 \pm 3.7$ & $29.5 \pm 1.5$ & * \\
\hline SDRR (ms) & $83.9 \pm 4.4$ & $44.3 \pm 1.6$ & $*$ & $51.9 \pm 2.5$ & $39.5 \pm 1.6$ & $*$ & $73.0 \pm 4.2$ & $50.2 \pm 2.1$ & $*$ \\
\hline $\mathrm{RR}(\mathrm{ms})$ & $951.6 \pm 20.6$ & $733.0 \pm 8.7$ & $*$ & $710.5 \pm 11.3$ & $622.2 \pm 7.4$ & $*$ & $833.8 \pm 19.3$ & $683.4 \pm 9.5$ & $*$ \\
\hline HFn & $0.44 \pm 0.01$ & $0.37 \pm 0.01$ & $*$ & $0.32 \pm 0.01$ & $0.31 \pm 0.01$ & 0.317 & $0.40 \pm 0.01$ & $0.37 \pm 0.01$ & 0.015 \\
\hline LFn & $0.38 \pm 0.01$ & $0.43 \pm 0.01$ & $*$ & $0.49 \pm 0.01$ & $0.49 \pm 0.01$ & 0.817 & $0.42 \pm 0.01$ & $0.43 \pm 0.01$ & 0.582 \\
\hline $\begin{array}{l}\text { Total Power } \\
\left(\mathrm{ms}^{2}\right)\end{array}$ & $146.0 \pm 4.4$ & $106.4 \pm 1.9$ & $*$ & $111.3 \pm 2.3$ & $104.2 \pm 1.9$ & 0.014 & $121.9 \pm 3.2$ & $99.3 \pm 1.7$ & $*$ \\
\hline $\mathrm{HF}\left(\mathrm{ms}^{2}\right)$ & $14.9 \pm 1.1$ & $6.9 \pm 0.4$ & $*$ & $5.7 \pm 0.3$ & $4.5 \pm 0.2$ & 0.002 & $10.5 \pm 0.7$ & $7.0 \pm 0.4$ & $*$ \\
\hline $\operatorname{LF}\left(\mathrm{ms}^{2}\right)$ & $12.5 \pm 0.8$ & $7.8 \pm 0.3$ & $*$ & $8.9 \pm 0.5$ & $7.0 \pm 0.3$ & $*$ & $11.0 \pm 0.8$ & $7.9 \pm 0.4$ & $*$ \\
\hline $\operatorname{VLF}\left(\mathrm{ms}^{2}\right)$ & $112.4 \pm 2.7$ & $88.1 \pm 1.6$ & $*$ & $93.4 \pm 1.9$ & $89.3 \pm 1.6$ & 0.073 & $95.7 \pm 2.1$ & $80.7 \pm 1.1$ & $*$ \\
\hline
\end{tabular}

$* p<5 \times 10^{-4}$

\section{Discussion}

We measured cardiac performance indices continuously during a protocol consisting of various standardised physiological states, thus providing a comprehensive characterisation of cardiovascular and haemodynamic responses to 'early' pregnancy. The main findings were that, compared with non-pregnant values, early pregnancy is associated with (1) increased heart rate and cardiac output in all physiological states, (2) decreased stroke volume but only in the supine position, (3) shortening of QTe and QTa intervals in all physiological states, (4) increases in QTeVI and QTaVI in all physiological states, (5) increases in RMSSDQTe and SDQTe only in the supine position, and (6) reductions in RMSSD, SDRR, and HFn in all states.

Previous studies have strongly debated the time course of haemodynamic changes during early pregnancy (Capeless \& Clapp, 1989; Duvekot et al., 1993). We therefore also measured haemodynamic changes, allowing us to characterise the influence of our protocol on conventional measures of cardiovascular performance. We observed (1) an increase in heart rate and a decrease in stroke volume in the supine position (suggesting that at this stage of pregnancy heart rate is the predominant mechanism for increasing cardiac output), (2) a reduction in total peripheral resistance (TPR) during pregnancy in all states, (3) a reduction in end diastolic volume index (EDI) during the supine posture and in response to 
metronomic breathing, (4) a reduction in left ventricular ejection time (LVET) in the supine posture and exercise state, and (5) a reduction in blood pressure in pregnancy but only during metronomic breathing. Consistent with our findings, Duvekot et al. (1993) found an increase in cardiac output, caused by a rise in heart rate, by the tenth week of gestation. Similarly, Capeless \& Clapp (1989) found a rise in cardiac output by 8 weeks gestation in comparison to pre-conception values; however this rise was predominantly as a result of increased stroke volume as opposed to heart rate. In contrast to our results Mahendru et al. (2014) found no increase in cardiac output, despite an increase in heart rate at 6 weeks gestation. The early time point at which this measurement was taken may be the reason for this difference. Our observation of a reduction in TPR is also consistent with other authors (Duvekot et al., 1993; San-Frutos et al., 2011). The reduction in blood pressure observed during metronomic breathing may be associated with a reduction in parasympathetic activity with pregnancy.

Baumert at al. (2010) have previously found that QT interval is shortened by the second half of pregnancy (post 28 weeks) in comparison to non-pregnant controls but they found no changes at earlier gestational ages. These authors also observed that rate-corrected QT (QTc) was not changed by pregnancy and so concluded that the duration of ventricular repolarisation is entirely rate-dependent. Gondoni et al. (2011) found a strong relationship between ejection fraction and QTc and since it is well documented that ejection fraction is unaltered with pregnancy (Capeless \& Clapp, 1989), this may explain the unaltered QTc with gestation. We have extended these observations by showing that uncorrected QTe and QTa are significantly reduced even by the end of the first trimester and notably in all physiological states. This suggests that pregnant women are able to maintain cardiac repolarisation at a safe level, during rest and light exercise, without exposing them to high risk arrhythmia. We did not separately assess rate-corrected QT as our objective here was to examine QT variability and (using QTVI) the relative magnitudes of QT and RR variabilities.

The HRV indices RMSSD, SDRR and HFn were all reduced during pregnancy, whilst LFn increased. Notably HFn was reduced in both the supine and metronomic breathing states, both of which are associated with dominant parasympathetic control. Thus these changes in HRV appear to reflect a substantial reduction in parasympathetic tone and an increase in sympathetic activity by the end of the first trimester of pregnancy. Sympathetic activity has 
previously been noted to increase by late pregnancy (measured using HRV) (Speranza et al., 1998; Kuo et al., 2000; Voss et al., 2000), with sympathetic hyperactivity at this stage indicating an increased risk of hypertension and pre-eclampsia (Greenwood et al., 2003) but its relative influence during early pregnancy has not been convincingly determined. Jarvis et al. (2012) observed marked increases in systemic sympathetic neural activity within the first weeks of conception (via muscle sympathetic neural activation). Conversely, Kuo et al. (1997) reported a higher vagal and a lower sympathetic modulation in three different recumbent positions (in particular the supine position) during pregnancy. An increase in baseline (supine) sympathetic activity during the early stages of pregnancy might have advantageous effects as it enhances the ability of pregnant women to adapt to haemodynamic changes and thus reduces the risk of orthostatic hypotension upon standing. Interestingly, LFn (a surrogate measure of sympathetic activity) was the same for both pregnant and non-pregnant women during light exercise, despite a further increase in cardiac output in the pregnant participants. This suggests that light exercise poses no increased risk to women during the early stages of pregnancy. Further longitudinal characterisations of HRV are therefore required to clarify the dynamics of cardiac autonomic control during pregnancy.

Whether these observed alterations in autonomic control of heart rate (supraventricular influences) also influence ventricular myocardial repolarisation (i.e. QT interval duration and variability) is yet to be clearly determined. Myocardial repolarisation might be influenced by pathological, physiological or other structural changes in the heart, or by autonomic neural activity changes (Piccirillo et al., 2007). Using an analogous approach to the time-domain assessment of HRV we assessed QT variability in terms of two statistical measures: RMSSDQTe and SDQTe. We found that in the supine position QT variability was increased by the end of the first trimester in comparison to non-pregnant controls, whilst no changes were seen during exercise or metronomic breathing states. It might be speculated that the autonomic nervous system could influence ventricular repolarisation independently of the sino-atrial node, considering the observed reduction in parasympathetic activity during the first trimester, as studies have shown parasympathetic withdrawal to have an important influence on the shortening of the QT interval at higher heart rates during exercise (Magnano et al., 2002) and therefore the same mechanism might be relevant to the 
physiological changes during pregnancy. However ventricular repolarisation is thought to be predominantly controlled by the beta-adrenergic system and any vagal effects are indirect and secondary to changes in heart rate (Can et al., 2002). Advancing gestation is also associated with hypervolaemia, leading to structural changes in the left ventricle. These changes may alter specific regions and layers of the ventricular tissue thus altering ion channel activity.

The QT variability index (QTVI), first proposed by Berger et al. (1997), describes the relative magnitude of temporal variability in myocardial depolarisation and repolarisation phases. It has been suggested that QTVI can be used to quantify the relative autonomic influences on the atrial and ventricular myocardium during rest and exercise, and that it might reflect the balance between heart rate-dependent and heart rate-independent influences on the QT interval (Lewis \& Short, 2008). Previously an increase in QTVI has been associated with a greater susceptibility to ventricular arrhythmias and sudden death (Berger et al., 1997; Atiga et al., 2000). However, ours is the first study to investigate the influence of pregnancy on QTVI. We found that QTVI was elevated as a result of early physiological changes associated with pregnancy in all physiological states. QTVI was highest during exercise in both the pregnant and non-pregnant women, further increased in the pregnant women. This increase in QTVI suggests that QT variability is more predominant over changes in RR variability at this time, indicative of a parasympathetic shift in the sympatho-vagal balance with pregnancy. As pregnancy is associated with an increase in arrhythmogenic events (Siu et al., 2001; Nakagawa et al., 2004; Li et al., 2008) the use of QTVI as a potential biomarker for screening patients at such an early stage might be of great clinical importance. QTVI is known to be further increased with exercise (Lewis et al., 2006; Lewis \& Short, 2008). At moderate intensities of exercise Lewis et al. (2006) reported a progressive increase in QTVI with work rate. Healthy non-pregnant individuals typically have a QTVI value of around -1 or lower at rest: Dobson et al. (2013) reported a mean QTVI of -1.34 in 173 healthy volunteers (unpublished data). Consistent with Dobson et al. (2013) we noted non-pregnant values of 1.4 and -1.3 (for QTeVI and QTaVI respectively) but higher values of around -0.9 in pregnant women. Despite increased values with pregnancy, at no point did QTVI become positive or exceed a value of 0.1 , a value proposed as a discriminator for higher risk of arrhythmogenic events (Atiga et al., 1998). This indicates that QT variability is not markedly influenced by 
pregnancy during the first trimester, suggesting that it is unlikely to be the cause of the increased risk of arrhythmia in pregnancy. It remains to be seen whether QTVI increases further during later gestation, and more work is needed to evaluate whether QT variability might be the cause of increased arrhythmia susceptibility during that stage of pregnancy. Our study has shown that QTVI is a sensitive marker of not only physiological state but also of gestational age and therefore its use to determine arrhythmogenic events in normal and abnormal pregnancy should be considered. Using QTVI as a marker of arrhythmogenic activity during pregnancy might enable non-invasive assessment of the need for antiarrhythmic treatment as well as quantifying the influence of lifestyle factors such as exercise on cardiac electrical function.

\section{Limitations}

We used late first trimester/early second trimester values as a baseline measure to characterise the extent of cardiovascular changes within 'early' pregnancy. Ideally we would have recruited women prior to conception because it is known that physiological changes are evident as early as the sixth week of gestation, and this would have enabled a true assessment of 'early' pregnancy changes. However, owing to time constraints and a specific period of funding for our work this was not feasible in the present study. We did not assess heart rate-corrected QT and were thus not able to comment on the likely determinants of QT interval shortening at this early stage of pregnancy.

\section{Conclusion}

The early stages of pregnancy are associated with substantial changes in cardiac autonomic control, with reductions in parasympathetic tone and increases in sympathetic activity. Whether these changes influence ventricular myocardial repolarisation and thus predispose women to arrhythmogenic events is yet to be determined. We have shown that QTVI is a sensitive marker of not only physiological state but also of gestational age. The use of QTVI as a marker of arrhythmia risk during pregnancy is feasible and could provide clinically important information regarding the need for prophylactic anti-arrhythmia treatment. We 
have used unique and novel concepts to assess cardiovascular responses to early pregnancy. These non-invasive methods have great clinical potential in predicting and identifying normal and abnormal responses to increased physiological stress, such as occur during orthostatic manoeuvre and light intensity exercise.

Declaration of Interest

The authors report no conflicts of interest. The authors alone are responsible for the content and writing of the paper. R Carpenter received a NISCHR (Welsh Government National Institute for Social Care and Health Research) PhD studentship, and The Cooperative Pharmacy (UK) provided financial support for project consumables. 


\section{References}

Adamson, D. L., \& Nelson-Piercy, C. (2007). Managing palpitations and arrhythmias during pregnancy. Heart, 93(12), 1630-1636. doi: 10.1136/hrt.2006.098822

Atiga, W. L., Calkins, H., Lawrence, J. H., Tomaselli, G. F., Smith, J. M., \& Berger, R. D. (1998). Beat-tobeat repolarization lability identifies patients at risk for sudden cardiac death. J Cardiovasc Electrophysiol, 9(9), 899-908.

Atiga, W. L., Fananapazir, L., McAreavey, D., Calkins, H., \& Berger, R. D. (2000). Temporal repolarization lability in hypertrophic cardiomyopathy caused by beta-myosin heavy-chain gene mutations. Circulation, 101(11), 1237-1242.

Baumert, M., Seeck, A., Faber, R., Nalivaiko, E., \& Voss, A. (2010). Longitudinal changes in QT interval variability and rate adaptation in pregnancies with normal and abnormal uterine perfusion. Hypertens Res, 33(6), 555-560. doi: 10.1038/hr.2010.30

Berger, R. D., Kasper, E. K., Baughman, K. L., Marban, E., Calkins, H., \& Tomaselli, G. F. (1997). Beatto-beat QT interval variability: novel evidence for repolarization lability in ischemic and nonischemic dilated cardiomyopathy. Circulation, 96(5), 1557-1565.

Can, I., Aytemir, K., Köse, S., \& Oto, A. (2002). Physiological mechanisms influencing cardiac repolarization and QT interval. Card Electrophysiol Rev, 6(3), 278-281.

Capeless, E. L., \& Clapp, J. F. (1989). Cardiovascular changes in early phase of pregnancy. Am J Obstet Gynecol, 161(6 Pt 1), 1449-1453.

Chamchad, D., Horrow, J. C., Nakhamchik, L., \& Arkoosh, V. A. (2007). Heart rate variability changes during pregnancy: an observational study. Int J Obstet Anesth, 16(2), 106-109. doi: 10.1016/j.ijoa.2006.08.008

D'Silva, L. A., Davies, R. E., Emery, S. J., \& Lewis, M. J. (2014). Influence of somatic state on cardiovascular measurements in pregnancy. Physiol Meas, 35(1), 15-29. doi: 10.1088/0967$3334 / 35 / 1 / 15$

Dobson, C. P., Kim, A., \& Haigney, M. (2013). QT Variability Index. Prog Cardiovasc Dis, 56(2), 186194. doi: 10.1016/j.pcad.2013.07.004

Duvekot, J. J., Cheriex, E. C., Pieters, F. A., Menheere, P. P., \& Peeters, L. H. (1993). Early pregnancy changes in hemodynamics and volume homeostasis are consecutive adjustments triggered by a primary fall in systemic vascular tone. Am J Obstet Gynecol, 169(6), 1382-1392.

Gondoni, L. A., Titon, A. M., Montano, M., Caetani, G., Nibbio, F., \& Schwartz, P. J. (2011). The myth of QT shortening by weight loss and physical training in obese subjects with coronary heart disease. Obesity (Silver Spring), 19(1), 200-203. doi: 10.1038/oby.2010.132

Greenwood, J. P., Scott, E. M., Walker, J. J., Stoker, J. B., \& Mary, D. A. (2003). The magnitude of sympathetic hyperactivity in pregnancy-induced hypertension and preeclampsia. Am J Hypertens, 16(3), 194-199.

Haigney, M. C., Zareba, W., Gentlesk, P. J., Goldstein, R. E., Illovsky, M., McNitt, S., . . investigators, M. A. D. I. T. I. (2004). QT interval variability and spontaneous ventricular tachycardia or fibrillation in the Multicenter Automatic Defibrillator Implantation Trial (MADIT) II patients. J Am Coll Cardiol, 44(7), 1481-1487. doi: 10.1016/j.jacc.2004.06.063

Jarvis, S. S., Shibata, S., Bivens, T. B., Okada, Y., Casey, B. M., Levine, B. D., \& Fu, Q. (2012). Sympathetic activation during early pregnancy in humans. J Physiol, 590(Pt 15), 3535-3543. doi: 10.1113/jphysiol.2012.228262

Jose, A. D., \& Collison, D. (1970). The normal range and determinants of the intrinsic heart rate in man. Cardiovasc Res, 4(2), 160-167.

Kanoupakis, E. M., \& Vardas, P. E. (2005). Arrhythmias and pregnancy. Hellenic J Cardiol, 46(5), 317319.

Kron, J., \& Conti, J. B. (2007). Arrhythmias in the pregnant patient: current concepts in evaluation and management. J Interv Card Electrophysiol, 19(2), 95-107. doi: 10.1007/s10840-0079139-4 
Kuo, C. D., Chen, G. Y., Yang, M. J., Lo, H. M., \& Tsai, Y. S. (2000). Biphasic changes in autonomic nervous activity during pregnancy. Br J Anaesth, 84(3), 323-329.

Kuo, C. D., Chen, G. Y., Yang, M. J., \& Tsai, Y. S. (1997). The effect of position on autonomic nervous activity in late pregnancy. Anaesthesia, 52(12), 1161-1165.

Lewis, M. J., Rassi, D., \& Short, A. L. (2006). Analysis of the QT interval and its variability in healthy adults during rest and exercise. Physiol Meas, 27(11), 1211-1226. doi: 10.1088/0967$3334 / 27 / 11 / 013$

Lewis, M. J., \& Short, A. L. (2008). Relationship between electrocardiographic RR and QT interval variabilities and indices of ventricular function in healthy subjects. Physiol Meas, 29(1), 1-13. doi: 10.1088/0967-3334/29/1/001

Li, J. M., Nguyen, C., Joglar, J. A., Hamdan, M. H., \& Page, R. L. (2008). Frequency and outcome of arrhythmias complicating admission during pregnancy: experience from a high-volume and ethnically-diverse obstetric service. Clin Cardiol, 31(11), 538-541. doi: 10.1002/clc.20326

Magnano, A. R., Holleran, S., Ramakrishnan, R., Reiffel, J. A., \& Bloomfield, D. M. (2002). Autonomic nervous system influences on QT interval in normal subjects. J Am Coll Cardiol, 39(11), 18201826.

Mahendru, A. A., Everett, T. R., Wilkinson, I. B., Lees, C. C., \& McEniery, C. M. (2014). A longitudinal study of maternal cardiovascular function from preconception to the postpartum period. $J$ Hypertens, 32(4), 849-856. doi: 10.1097/HJH.0000000000000090

McNarry, M. A., \& Lewis, M. J. (2012). Heart rate variability reproducibility during exercise. Physiol Meas, 33(7), 1123-1133. doi: 10.1088/0967-3334/33/7/1123

Molnar, J., Zhang, F., Weiss, J., Ehlert, F. A., \& Rosenthal, J. E. (1996). Diurnal pattern of QTc interval: how long is prolonged? Possible relation to circadian triggers of cardiovascular events. J Am Coll Cardiol, 27(1), 76-83. doi: 10.1016/0735-1097(95)00426-2

Nakagawa, M., Katou, S., Ichinose, M., Nobe, S., Yonemochi, H., Miyakawa, I., \& Saikawa, T. (2004). Characteristics of new-onset ventricular arrhythmias in pregnancy. J Electrocardiol, 37(1), 47-53.

Piccirillo, G., Magrì, D., Matera, S., Magnanti, M., Torrini, A., Pasquazzi, E., . . Barillà, F. (2007). QT variability strongly predicts sudden cardiac death in asymptomatic subjects with mild or moderate left ventricular systolic dysfunction: a prospective study. Eur Heart J, 28(11), 13441350. doi: 10.1093/eurheartj/ehl367

Porter, M. J., Morton, J. B., Denman, R., Lin, A. C., Tierney, S., Santucci, P. A., . . Wilber, D. J. (2004). Influence of age and gender on the mechanism of supraventricular tachycardia. Heart Rhythm, 1(4), 393-396. doi: 10.1016/j.hrthm.2004.05.007

San-Frutos, L., Engels, V., Zapardiel, I., Perez-Medina, T., Almagro-Martinez, J., Fernandez, R., \& BajoArenas, J. M. (2011). Hemodynamic changes during pregnancy and postpartum: a prospective study using thoracic electrical bioimpedance. J Matern Fetal Neonatal Med, 24(11), 1333-1340. doi: 10.3109/14767058.2011.556203

Shotan, A., Ostrzega, E., Mehra, A., Johnson, J. V., \& Elkayam, U. (1997). Incidence of arrhythmias in normal pregnancy and relation to palpitations, dizziness, and syncope. Am J Cardiol, 79(8), 1061-1064.

Speranza, G., Verlato, G., \& Albiero, A. (1998). Autonomic changes during pregnancy: assessment by spectral heart rate variability analysis. J Electrocardiol, 31(2), 101-109.

Voss, A., Malberg, H., Schumann, A., Wessel, N., Walther, T., Stepan, H., \& Faber, R. (2000). Baroreflex sensitivity, heart rate, and blood pressure variability in normal pregnancy. Am J Hypertens, 13(11), 1218-1225. 